\title{
Synaptonemal complex analysis of reciprocal chromosome translocations in the domestic pig
}

\author{
DAF Villagomez ${ }^{1}$, I Gustavsson ${ }^{1}$, L Plöen $^{2}$ \\ ${ }^{1}$ Swedish University of Agricultural Sciences, \\ Department of Animal Breeding and Genetics; and \\ ${ }^{2}$ Department of Anatomy and Histology, Uppsala, Sweden
}

(Proceedings of the 9th European Colloquium on Cytogenetics of Domestic Animals; Toulouse-Auzeville, 10-13 July 1990)

synaptonemal complex analysis / reciprocal chromosome translocation / domestic pig

One main feature of chromosome translocations in man and mouse is their common association with meiotic impairment of the carriers. This happens especially when acrocentric chromosomes are involved and unpaired segments of the translocation configuration are associated with the sex bivalent (Chandley, 1982; Forejt, 1982). In the domestic pig, there is only one indication that reciprocal translocations could lead to meiotic degenerations (Gustavsson et al, 1990). We describe here the male synaptonemal complex (SC) behavior, evaluated by electron microscopy and prepared according to Counce and Meyer (1973), of two reciprocal translocations: $\operatorname{rcp}(1 q+/ 18 q-)$ and $\operatorname{rcp}(7 q+/ 17 q-)$. Both translocations resulted in one extremely small derivative chromosome (fig 1) and the breakage/rejoining points of the involved chromosomes were localized proximally in the telocentric chromosomes and terminally in the $\mathrm{q}$ arm of the biarmed chromosomes.

Both boars were of the Hampshire breed. The boar with $\mathrm{rcp}(1 \mathrm{q}+/ 18 \mathrm{q}-)$ had a litter size decreased by $45 \%$ in 15 litters, but had a normal semen picture and no repeat breeding; there was one stillborn piglet. The boar carrying the rcp $(7 \mathrm{q}+/ 17 \mathrm{q}-)$ also had a normal semen picture but the breeding records showed a reduction of $41 \%$ in the litter size along with 3 repeat breedings. Approximately $9 \%$ of the liveborn piglets died early and most of them appeared to be malformed. Cytogenetic investigations showed that some of the malformed piglets were tertiary monosomics. 


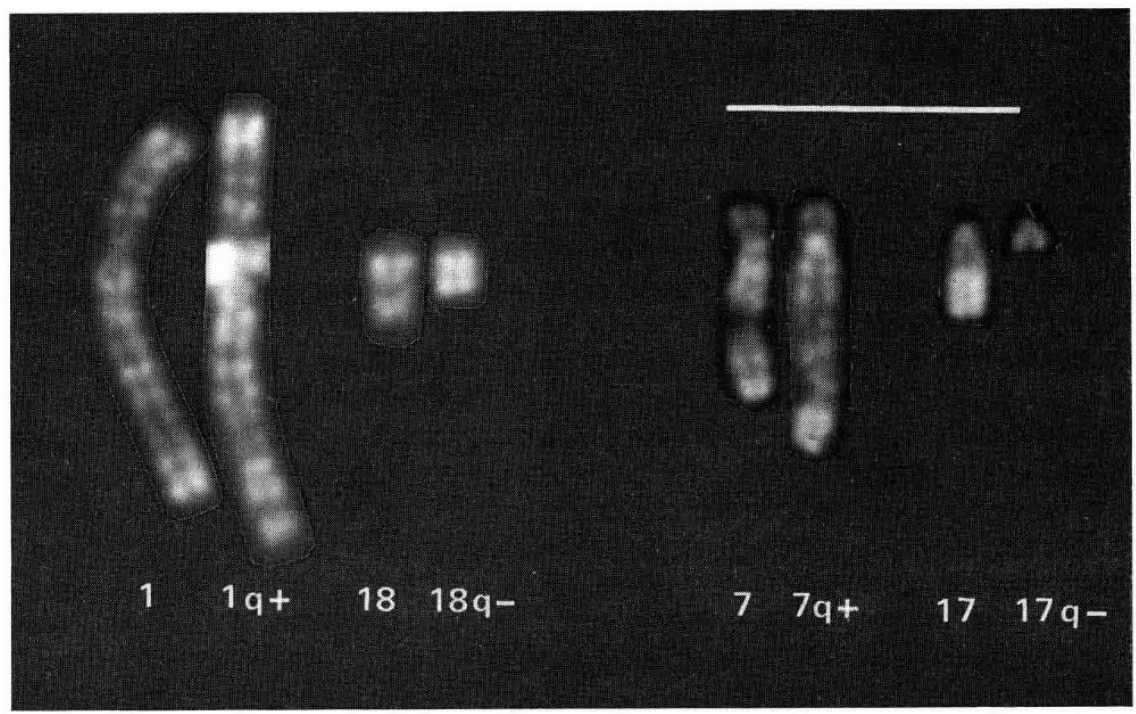

Fig 1. RBA-banded translocation chromosomes of $\operatorname{rcp}(1 \mathrm{q}+/ 18 \mathrm{q}-)$ and $\operatorname{rcp}(7 \mathrm{q}+/ 17 \mathrm{q}-)$. Bar indicates $10 \mu \mathrm{m}$.

\section{$\operatorname{rcp}(1 q+/ 18 q-)$}

Fifty-seven cells were investigated at the pachytene stage. With the exception of one spermatocyte with two heteromorphic bivalents, the remaining 56 cells demonstrated a quadrivalent. The most frequent type of configuration ( 2 early pachytene, 12 mid-pachytene and 12 late pachytene cells) was a completely paired cross-shaped quadrivalent morphology, which involved some non-homologous pairing of chromosomes 1 and 18. Completely paired quadrivalents without distinctive heterosynapsis were found at mid- and late pachytene ( 3 and 2 cells, respectively). This kind of quadrivalent probably reflects the highest possible degree of homologous pairing (fig 2a). Another common type of morphology observed was the buckle configuration, which could be classified into two categories. One category of buckle quadrivalent ( 3 cells at mid- and 13 cells at late pachytene) showed remnants of central elements in the interstitial unpaired region. The other type of buckle quadrivalent was without remnants of central elements in the interstitially unpaired segments ( 2 carly, 1 mid- and 4 late pachytene cells). Finaliy, in two cells at mid-pachytene, the quadrivalent had the axes of the telocentric chromosomes completely unpaired. The free ends were end-to-end associated with an autosomal bivalent.

$$
\operatorname{rcp}(7 q+/ 17 q-)
$$

The synaptic behavior was analyzed in a total of 64 cells. Seven spermatocytes $(10.9 \%)$ were in the zygotene stage. In 4 of these cells, the translocation configuration consisted of an open trivalent plus a univalent (fig $2 \mathrm{~b}$ ). This type of trivalent 


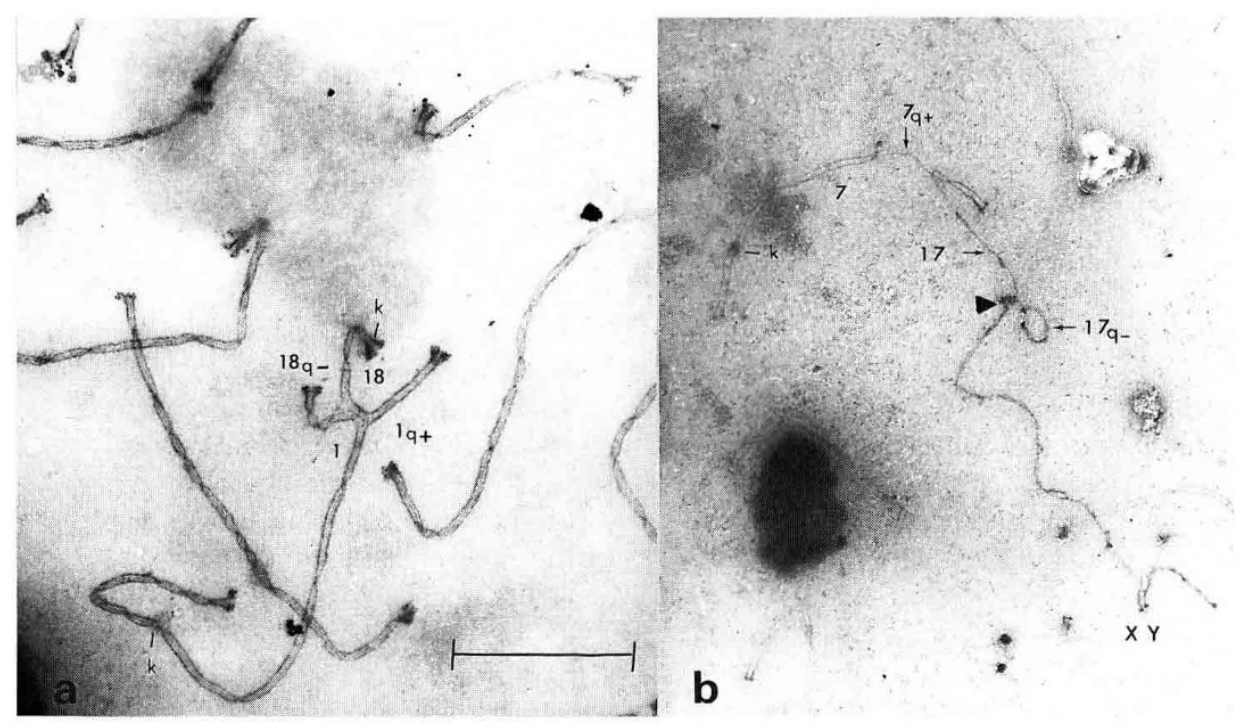

Fig 2. a. SC of completely paired quadrivalent of the $\operatorname{rcp}(1 \mathrm{q}+/ 18 \mathrm{q}-)$. The telocentric bivalents are associated by their kinetochores to a chromocenter. $\mathbf{b}$. SC trivalent + univalent of the $\operatorname{rcp}(7 q+/ 17 q-)$. Notice the pairing between the sex bivalent and the translocation configuration (arrowhead). The kinetochores (k) are indicated. The same bar indicates approximately $3.5 \mu \mathrm{m}$ in $\mathbf{a}$ and $5.4 \mu \mathrm{m}$ in $\mathbf{b}$.

occurred when the axis of the derivative chromosome 17 was dissociated and when there was no heterosynapsis between the normal chromosomes 7 and 17. A univalent, ring-shaped ( 1 cell) and rod-shaped ( 2 cells), was observed in 3 of the 4 cells having an open trivalent. In the 3 remaining zygotene cells, 2 types of quadrivalent configurations were found. An open quadrivalent, with the axis of chromosome $17 q-$ paired either with chromosome number 7 or 17 , was present in 2 cells, and in one cell chromosome $17 q$ - was partially synapsed with the 2 normal chromosomes, forming a buckle quadrivalent configuration. At pachytene, a variety of configurations were formed. Fourty-seven (82.5\%) out of 57 cells showed a quadrivalent configuration, of which only $11(23.4 \%)$ had the quadrivalent completely paired. Twenty-three (48.9\%) spermatocytes with a quadrivalent showed an open configuration, which could be classified into 2 types: chromosome $17 q-$ synapsed with chromosome 17 ( 7 cells), and chromosome $17 q$ - synapsed with chromosome 7 (16 cells). In both types, chromosomes 17 and $7 q+$ were unpaired to some extent, which might be due to non-homology. As expected, most of chromosome 7 paired with the homologous segment of chromosome $7 \mathrm{q}+$, but also showed a short segment available for pairing with chromosome $17 q-$. Buckle configuration was found in 13 spermatocytes, which may result from either incomplete pairing in the interstitial regions of the quadrivalent at early pachytene or desynapsis at mid-late pachytene starting in these regions of completely paired quadrivalents. Eight cells had completely paired trivalents plus a univalent (axis of chromosome 17q-) but only 2 spermatocytes exhibited an open trivalent plus the axis of chromosome $17 \mathrm{q}-$. The sex bivalent was paired end-to-end with translocation configurations in 12 cells (18.7\%). 
There was a clear difference in the pairing behavior of the two translocations. The $\operatorname{rcp}(1 \mathrm{q}+/ 18 \mathrm{q}-)$ showed, as previously reported for several translocations in boars (Gustavsson et al, 1988; Gabriel-Robez et al, 1988), a regular synaptic behavior. Quadrivalent configurations were most often found as well in spermatocytes of the boar carrying the $\operatorname{rcp}(7 q+/ 17 q-)$, but many cells demonstrated dissociations. In both translocations, completely homologously paired quadrivalents were less frequent than any other class of configurations. This may be due to mechanical problems of pairing. Another possible explanation is that heterologous pairing between the normal chromosomes involved in each translocation starts at the same time as homologous pairing of the translocation chromosomes. This is supported by the fact that, in both translocations, buckle quadrivalents were seen most frequently at the latest stages of pachytene; some of them showed remnants of central elements. Thus, non-homologous pairing between the normal chromosomes could begin at early pachytene followed by desynapsis at mid-pachytene not being substituted by homologous pairing. If the Miklos (1974) hypothesis applies in the case of reciprocal translocations in boars, early heterosynapsis saturating the pairing sites would be a mechanism to avoid germ cell loss.

The common occurrence of trivalent plus univalent $(20.9 \%)$ in the $\operatorname{rcp}(7 q+/ 17 q-)$ boar suggests that the derivative element often was not bound by a chiasma. It is interesting to note that the open quadrivalents showed more dissociations of chromosome $17 \mathrm{q}$ - from chromosome 17 than from chromosome 7 (17 and 8 cells, respectively). This finding could be explained by chiasmata formed more often telomerically (segment pairing with chromosome 7) than centromerically (segment pairing with chromosome 17), or that pairing of chromosome $17 q-$ begins preferably at its distal end. Preliminary findings in a tertiary trisomic boar have shown that the chromosome $17 q$ - participates in a considerable number of trivalent formations, most of which are due to pairing with chromosome pair 7 . Another difference observed between the two translocations was the number of associations with the sex bivalent. In the $\operatorname{rcp}(7 q+/ 17 q-)$ the translocation configuration was often paired or associated with the sex bivalent $(17.1 \%)$, whereas no pairings with the sex bivalent were seen in the $\operatorname{rcp}(1 \mathrm{q}+/ 18 \mathrm{q}-)$. According to Lifschytz and Lindsley (1972), association of unpaired autosomal segments with the sex chromosomes can interfere with the X-inactivation which leads to breakdown of spermatogenesis. It should, however, be noted that both carriers demonstrated a normal semen picture and normal testicular histology.

\section{REFERENCES}

Chandley AC (1982) Normal and abnormal meiosis in man and other mammals. In: Serono Clinical Colloquium on Reproduction. (Crosignani PG et al, eds) Academic Press/Grune \& Stratton

Counce SJ, Meyer GF (1973) Differentiation of the synaptonemal complex and the kinetochore in Locusta spermatocytes studied by whole mount electron microscopy. Chromosoma 44, 231-253

Forejt J (1982) XY involvement in male sterility caused by autosome translocations - a hypothesis. In: Serono Clinical Colloquium on Reproduction (Crosignani PG et al, eds) Academic Press/Grune \& Stratton 
Gabriel-Robez O, Jafar H, Ratonponirina C, Boscher J, Bonneau J, Popescu CP, Rumpler $Y$ (1988) Heterosynapsis in a heterozygous fertile boar carrier of a $3 ; 7$ translocation. Chromosoma 97, 26-32

Gustavsson I, Świtoński M, Larsson K, Plöen L, Hojer K (1988) Chromosome-banding studies and synaptonemal complex analyses of four reciprocal translocations in the domestic pig. Hereditas 109, 169-184

Gustavsson I, Larsson K, Świtoński M, Plöen L (1990) Spontaneous chromosome translocation, $\mathrm{rcp}(2 ; 4)(\mathrm{p} 17 ; \mathrm{q} 11)$, in a boar demonstrating impaired gametogenesis and decreased litter size. Hereditas in press

Lifschytz E, Lindsey DL (1972) The role of X-chromosome inactivation during spermatogenesis. Proc Natl Acad Sci USA 69, 182-186

Miklos GLG (1974) Sex chromosome pairing and male fertility. Cytogenet Cell Genet 13, 558-577 